\title{
Robustness of railway rolling stock speed calculation using ground vibration measurements
}

\author{
Georges Kouroussis ${ }^{1, a}$, David P. Connolly ${ }^{2, b}$, Omar Laghrouche ${ }^{2, \mathrm{c}}$, Mike C. Forde ${ }^{3, \mathrm{~d}}$, Peter Woodward ${ }^{2, \mathrm{e}}$ \\ and Olivier Verlinden ${ }^{1, \mathrm{f}}$ \\ ${ }^{1}$ University of Mons, Department of Theoretical Mechanics, Dynamics and Vibrations, 31 Boulevard Dolez, \\ 7000 Mons, Belgium \\ ${ }^{2}$ Heriot-Watt University, Institute for Infrastructure \& Environment, Edinburgh EH14 4AS, UK \\ ${ }^{3}$ University of Edinburgh, Institute for Infrastructure and Environment, Alexander Graham Bell Building, \\ Edinburgh EH9 3JF, UK
}

\begin{abstract}
Evaluating railway vehicle speed is an important task for both railway operators and researchers working in the area of vehicle/track dynamics, noise and vibration assessment. The objective of this paper is to present a new technique capable of automatically calculating train speed from vibration sensors placed at short or long distances from the track structure. The procedure combines three separate signal processing techniques to provide high precision speed estimates. In order to present a complete validation, the robustness of the proposed method is evaluate using synthetic railway vibration time histories generated using a previously validated vibration numerical model. A series of simulations are performed, analysing the effect of vehicle speed, singular wheel and rail surface defects, and soil configuration. Virtual conditions of measurement are also examined, taking into account external sources other than trains, and sensor response. It is concluded that the proposed method offers high performance for several train/track/soil arrangements. It is also used to predict train speeds during field trials performed on operational railway lines in Belgium and in UK.
\end{abstract}

\section{Introduction}

Vehicle speed estimation is of growing interest in various railway applications. For example, many noise and vibrations phenomenons are associated to high-speed trains (HSTs). It is well known that high-speed railway networks are a competing mode of transportation to road and airplane traffic, for short, medium and long distances. Since 1981, when the French railway operator "Société Nationale de Chemin de fer Français" (SNCF) opened the first high-speed line between Paris and Lyon, the highspeed network has not stopped growing, and has become one of the largest and most valuable service networks in Europe. Even through the importance is lesser for lowspeed case, the vehicles speed in urban environments becomes a determining factor.

There are many sources of noise and vibration in the railway system, which strongly depends on the vehicle speed. For example, the rolling noise increases with the train speed $v_{0}$ at a rate of $30 \log _{10} v_{0}$ and the aerodynamic noise increases at a rate generally between $60 \log _{10} v_{0}$ and $80 \log _{10} v_{0}$ [1]. Other sources of railway noise, including

\footnotetext{
a e-mail: georges.kouroussis@umons.ac.be

b e-mail: d.connolly@hw.ac.uk

c e-mail: o.laghrouche@hw.ac.uk

d e-mail: m.forde@ed.ac.uk

e e-mail: p.k.woodward@hw.ac.uk

f e-mail: olivier.verlinden@umons.ac.be
}

traction noise or curve squeal noise, depends on the vehicle speed also.

In the last few years, several influential studies were conducted to analyse the potential of high-speed networks development on national economies. One of the concerns is the impact on the environment in terms of vibrations. Also a problematic issue is the so-called "supercritical phenomenon", which may appear when the vehicle's speed is close to the Rayleigh ground wave speed (the latest record is held by a TGV Sud-Est train, which reached $574.8 \mathrm{~km} / \mathrm{h}$ [2]). This phenomenon has been researched extensively. Theoretical and experimental studies of railway-induced, ground-borne vibrations have multiplied in the last twenty years. This has become evident because of the rapid development of these networks, particularly in Europe (Fig. 1). However, the interest of scientific and technical communities was renewed when abnormally high vibration amplitudes were recently recorded in Sweden [3]. The constraint on the peak velocity of high-speed trains also concerns the technical and safety limit imposed by the vehicle, as well as the track structure. For these reasons, it is important to mention the works of Degrande and Schillemans [4], Galvín and Domínguez [5], or Auersch [6]. The empirical model developed by Connolly et al. [7] outlined that it is possible to establish relationships between six key railway variables for ground vibration metrics: the vehicle speed is pointed out as one of these inputs. Picoux et al. demonstrated that the effect of train speed can be also perceived at low speeds (between 70 and $135 \mathrm{~km} / \mathrm{h}$ ) [8,9]. An accurate estimation

This is an Open Access article distributed under the terms of the Creative Commons Attribution License 2.0, which permits unrestricted use, distribution, and reproduction in any medium, provided the original work is properly cited. 


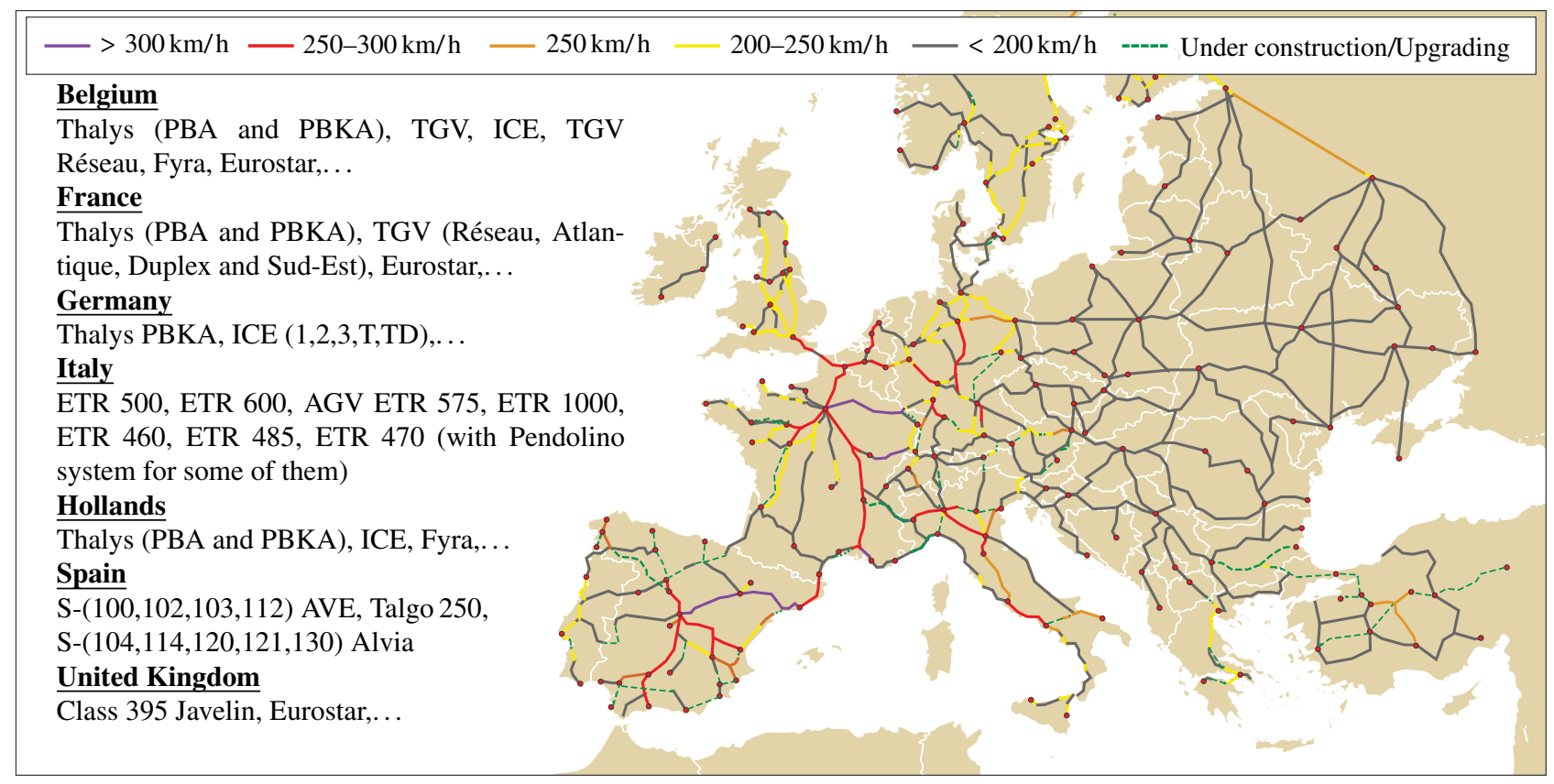

Figure 1. High-speed lines in Europe.

Table 1. Existing methods for evaluating vehicle speed.

\begin{tabular}{cclll}
\hline Method & Physical phenomenon & Advantage & Drawback & Error \\
\hline \hline tachometer & revolution-counter & $\begin{array}{l}\text { no additional measure- } \\
\text { ment }\end{array}$ & $\begin{array}{l}\text { administrative } \\
\text { procedure }\end{array}$ & $5 \%$ \\
\hline wheel counter & contact & very accurate & track access & $<1 \%$ \\
\hline radar & Doppler effect & Easy to use & $\begin{array}{l}\text { accuracy depending on } \\
\text { the position }\end{array}$ & from 1 to 5\% \\
\hline GPS & geolocalisation & cheap & using inside the train & from 5 to 10\% \\
\hline optic sensors & optic & very accurate & track access & $<1 \%$ \\
\hline camera & frame-counter & accessible device & track view access & $\begin{array}{l}\text { depending on } \\
\text { the camera } \\
\text { resolution }\end{array}$ \\
\hline
\end{tabular}

of the rolling stock speed is therefore unavoidable for the ground vibration topic.

Vehicle dynamics is strongly linked to the speed. Problems of ride quality, particularly in the lateral direction, become apparent at specific train speeds (a critical speed is also defined for this problem). Design of vehicle suspension is often investigated numerically [10] but a validation is generally supported by experiment. For simulation of such behaviour, the riding conditions, including vehicle speed, are necessary. The effect of rolling speed on the wheel/rail forces and track deterioration is also of great interest [11]; many investigation are been carried out, studying the speed coupling effect with slip, load, surface roughness, and water temperature.

All presented cases show the importance of estimating accurately train speed experimentally. This paper investigates the use of the dominant frequency method and defines its domain of validity. The vehicle speed $v_{0}$ is assumed to be constant in this work, a valid assumption because train acceleration/deceleration is relatively low. After a brief review of conventional train calculation methods, the procedure for train speed calculation using ground vibrations is recapitulated, with its various features.
The focus of this work is also to demonstrate the potential of this approach and its applicability to domestic and highspeed train configurations. Practical results are presented, based on numerical and experimental data.

\section{Assessment of train speed using the conventional methods}

A brief description of the possible techniques for monitoring train speed are shown in Table 1. The most common means is the direct use of the tachometer, but this makes it difficult to match an exact speed to each track section and furthermore requires good communication with the rolling stock manager. Wheel counters are commonly used to detect the passing of a train in lieu of the more common track circuit. Vehicle speed can be deduced from vehicle's geometry. Radar uses the Doppler effect. Its precision is high if the equipment is accurately positioned (near the track and exactly parallel to the rail). A GPS (global positioning system) is analogous to the tachometer with a improved estimation of the vehicle location. Despite this, for a large number of events, the cost can be prohibitive. By placing an optical photoelectric 


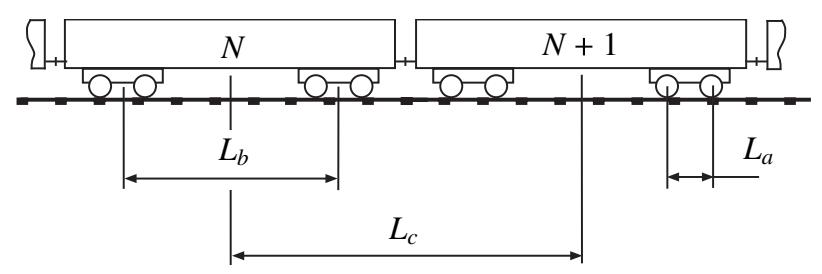

Figure 2. Main geometrical parameters of the train and the track.

device (with a transmitter and a receiver on both sides of the track), a method analogous to wheel counter can be used to evaluate the train speed in-situ, as proposed by $\mathrm{Ni}$ et al. [12]. If the railway landscape permits it (i.e. at-grade track or small backfill embankment), the use of a camera recorder, at large distance from the track, can provide the vehicle speed, based on the movie frame count. Fixed reference points on the video are necessary to enable accurate calculation (for example the portal catenary supports of the railroad line). This method has been used by Kouroussis et al. to assess HST's speed [13].

All these methods present some drawbacks. Most of them need a direct access to the track area or to the rail, which is not possible on every case due to economical and safety reasons.

\section{Assessment of train speed using the dominant frequency method}

As a first approximation, the passage of a train consists of a number of similar events, with certain delay times. If a single wheelset moving at speed $v_{0}$ is considered, its effect on the track can be represented by a series of impulse load regularly spaced according to the train dimensions (see Fig. 2 with $L_{a}$ the axle spacing, $L_{b}$ the bogie spacing and $L_{c}$ the carriage length). This phenomenon is visible in the frequency content of track deflection or soil loading with some points of interest.

- The fundamental axle and bogie passage frequencies, defined as $f_{a}=\frac{v_{0}}{L_{a}}$ and $f_{b}=\frac{v_{0}}{L_{b}}$, provide amplitude modulations at regular frequency intervals with zero amplitude at frequencies $\frac{2 k+1}{2} f_{b}$ and $\frac{2 k+1}{2} f_{a}(k \in \mathbb{N})$.

- The carriage passage frequencies

$$
f_{c}=n \frac{v_{0}}{L_{c}}(n \in \mathbb{N})
$$

impose the frequencies where the maximum amplitudes follow the aforementioned carriage envelope.

- The overall vibration magnitude decreases with frequency. The cut-off frequency is proportional to the foundation stiffness and to the vehicle speed. A vehicle running at high speed and/or on a track lying on a stiff foundation provides a large excitation frequency range.

Figure 3 presents the frequency spectra of the ballast vertical reaction. With the speed, each peak is located along a line with slope proportional to speed. Furthermore, it displays the fundamental $(n=1)$ and the harmonics $(n>1)$ of the carbody passage frequency directly in the excitation spectrum and as a function of the vehicle speed.

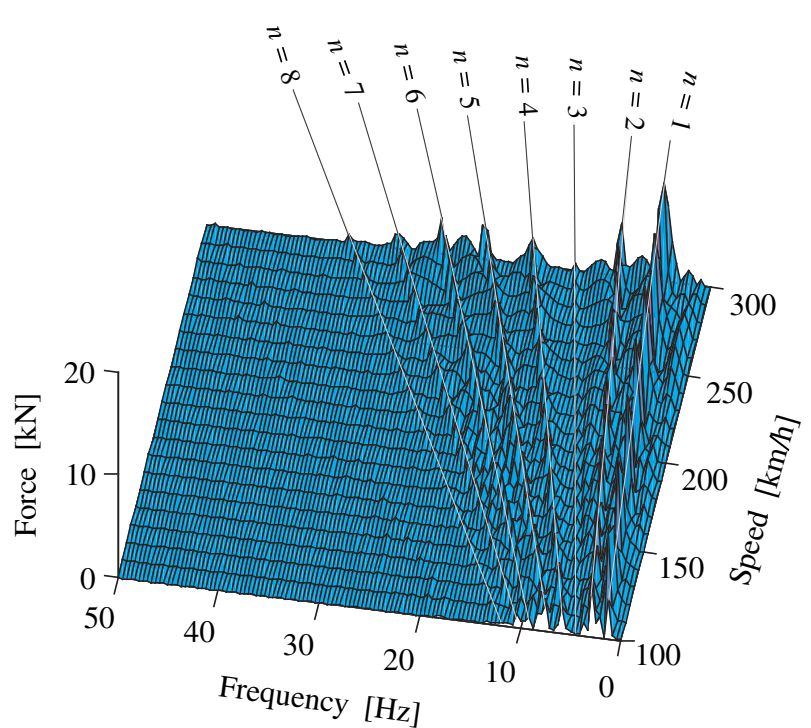

Figure 3. Spectral content of the soil loading as a function of the HST speed.

Some peaks exhibit high amplitudes (e.g. $n=1$ or 5 ) while others are almost completely suppressed $(n=3)$, due to the sidebands generated by the axle passage frequency. Overall, a decrease in amplitude with increasing frequency is also observable. These observations can be used to estimate vehicle speed and based the work on the dominant frequency method, initially proposed by $\mathrm{Ni}$ et al. [12] and enhanced in [14]. This method uses ground vibration signals $s_{i}(t)(i=1 \ldots m$, with $m$ the number of sensors $)$, since the same dominant frequencies intervene in the ground vibration spectra with the contribution of dynamic vehicle/track and soil interactions: as railway traffic generates ground vibration waves which induce small material deformations, the track and soil is considered as a linear mediums and can satisfy the superposition principle.

To pick these dominant frequencies, the fundamental excitation frequency must be estimated with precision. A cepstral analysis appears as a promising way to capture the aforementioned passage frequencies. It is defined as

$$
C_{s_{i}}(\tau)=\operatorname{DFT}\left(\log \left|\operatorname{DFT}\left(s_{i}(t)\right)\right|\right)
$$

using two successive discrete Fourier transforms (DFT) in order to obtain a "spectrum of a spectrum" as a function of quefrency $\tau$. The quefrency of the cepstrum peaks represents the modulation period, and its reciprocal, the modulating frequency. Although the cepstral transformation can be affected by several external factors (e.g. additional excitation mechanisms and signal noise), it gives a good estimation of the fundamental frequency by

$$
f_{c, 1}=\frac{v_{0}^{*}}{L_{c}}
$$

with $v_{0}^{*}$ the train speed evaluated as

$$
v_{0}^{*}=\frac{1}{3}\left(L_{c} f_{c}^{*}+L_{b} f_{b}^{*}+L_{a} f_{a}^{*}\right)
$$

where the cepstral characteristics $f_{a}^{*}, f_{b}^{*}$ and $f_{c}^{*}$ correspond to the excitation frequency associated to the 


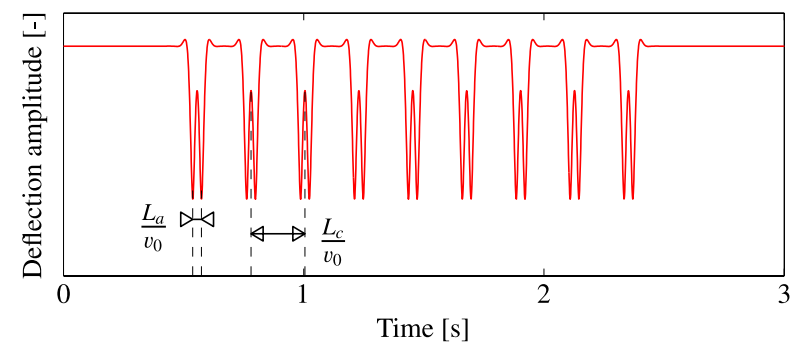

(a) Time history

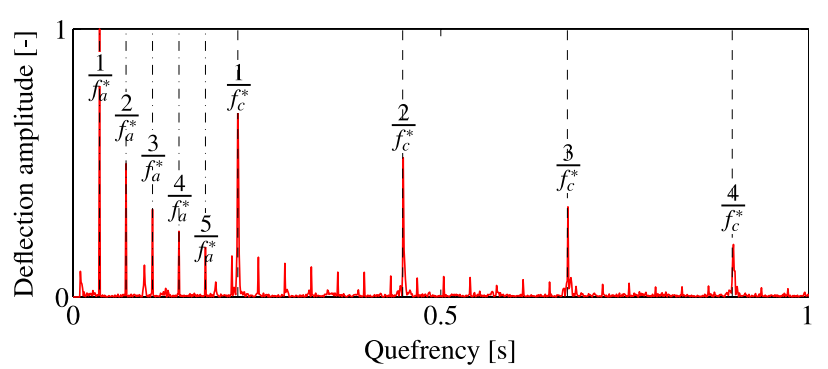

(b) Cepstrum

Figure 4. Application of the cepstral analysis to the track deflection corresponding to the passing of Thalys HST running at speed $v_{0}=300 \mathrm{~km} / \mathrm{h}$ (here, $f_{b}^{*}=f_{c}^{*}$ ).

wheelset periodicity, the bogie periodicity and the carriage periodicity, respectively. This calculation is illustrated in Fig. 4 where $f_{b}^{*}=f_{c}^{*}$ due to the specific configuration of Jacob's bogie equipping the Thalys HST.

The calculation starts from a frequency range $\left[f_{c, 1}^{(r 1)}\right.$; $\left.f_{c, 1}^{(r 2)}\right]$ specified by the user, within which, the maximum amplitude the fundamental frequency $f_{c, 1}$ are found using Eq. (3). Then a recursive calculation of the $n^{\text {th }}$ dominant frequency $f_{c, n}$ is performed by finding the maximum in the frequency range [12]:

$$
\left[f_{c, n}^{(r 1)} ; f_{c, n}^{(r 2)}\right]=\left[(n-0.45) \times f_{c, a v g} ;(n+0.45) \times f_{c, a v g}\right]
$$

where $f_{c, \text { avg }}$ represents the average of the fundamental frequency, calculated by [12]

$$
f_{c, a v g}=\frac{1}{n-1} \sum_{i=1}^{n-1} \frac{f_{c, i}}{i} \quad(n \geq 2) .
$$

For each dominant excitation frequency $f_{c, n}$, the train speed is estimated using

$$
v_{0, n}=\frac{f_{c, n} L_{c}}{n}
$$

and the final train speed is given by

$$
v_{0}=\min _{n}\left(v_{0, n+1}, v_{0, n}\right) \text {. }
$$

A safeguard can be implemented in the method in order to reject some poorly-estimated dominant excitation frequencies $f_{c, n}$ which do not satisfy the criterion [12]

$$
\left|\frac{f_{c, n}}{n}-f_{c, 1}\right|=0.3 f_{c, 1} .
$$

Additionally, $n$ is limited to 10 due to the decrease of amplitude with the distance (soil material damping).

The use of the dominant frequency method is improved by using the running rms instead of the original signal

$$
s_{i, r m s, \tau}\left(t_{0}\right)=\sqrt{\frac{1}{\tau} \int_{t_{0}-\tau}^{t_{0}} s_{i}^{2}(t) e^{\frac{t-t_{0}}{\tau}} \mathrm{d} t .}
$$

where $\tau$ is the integration constant time. This allows a clearer visualization of the passage of each wheelset.

To avoid limitations due to the signal frequency resolution and the other sources excitations [15], a regression analysis in the frequency domain is performed which combines the ground vibration measurements with an analytical solution to update the vehicle speed parameter. A simple formulation is used as a function of the frequency $f$ for the fitting, based on rules for time-delayed Dirac functions:

$$
\begin{aligned}
S^{t h}(f)= & A \mathrm{e}^{-\alpha f} \mid\left(1+\mathrm{e}^{-j 2 \pi f / f_{a}}\right)\left(1+\mathrm{e}^{-j 2 \pi f / f_{b}}\right) \\
& \left(1+\sum_{i=1}^{n_{c}} \mathrm{e}^{-j 2 \pi i f / f_{c}}\right) \mid
\end{aligned}
$$

where $n_{c}$ is the number of carriages and $A$ and $\alpha$ are the fitted amplitude and decay rate, respectively.

Figure 5 summarizes this procedure. The three speed estimation methods are combined and are successively used in order to increase prediction accuracy. If multiple ground vibration signals are used, an occurrence analysis is performed to estimate the speed mean $v_{0}$ from all $m$ sensors after each step. Additional details related to its development are available in [14].

\section{Speed calculation using numerically predicted results}

To ensure that the speed calculator model was capable of determining accurate vehicle speeds, it was validated using numerically predicted results [14]. This section completes this analysis by evaluating the robustness of the proposed method. Sets of results are created, in order to reproduce the different dynamic effects associated to the vehicle and to the conditions of measurement. A series of numerical time histories are used, considering a sufficient number of virtual sensors along a profile perpendicular to the track (Fig. 6) and measuring along the three directions $x, y$ and $z$ (for horizontal parallel to the track, horizontal perpendicular to the track or vertical, respectively).

To do it, the numerical prediction model based on a two-step approach and developed Kouroussis et al. [16] is used (Fig. 7). This model considers first the simulation of the vehicle/track/foundation subsystem, described by a system of second-order differential equations for the vehicle

$$
\left.\mathrm{M}_{v} \underline{\ddot{\mathbf{q}}}_{v}+\underline{\mathbf{h}} \underline{\mathbf{q}}_{v}, \underline{\mathbf{q}}_{v}, t\right)=0
$$

coupled to the equations of motion of the track

$$
\mathbf{M}_{t} \underline{\ddot{\mathbf{q}}}_{t}+\mathbf{C}_{t} \underline{\dot{\mathbf{q}}}_{t}+\mathbf{K}_{t} \underline{\mathbf{q}}_{t}=\underline{\mathbf{f}}_{t}
$$




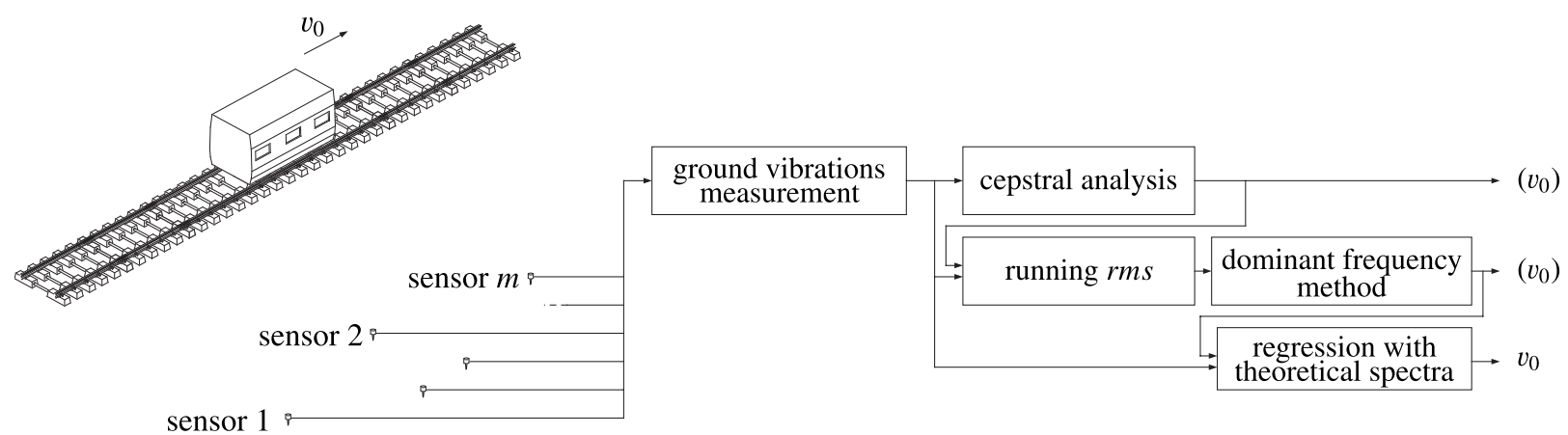

Figure 5. Chart of the automatic procedure for estimating the vehicle speed.

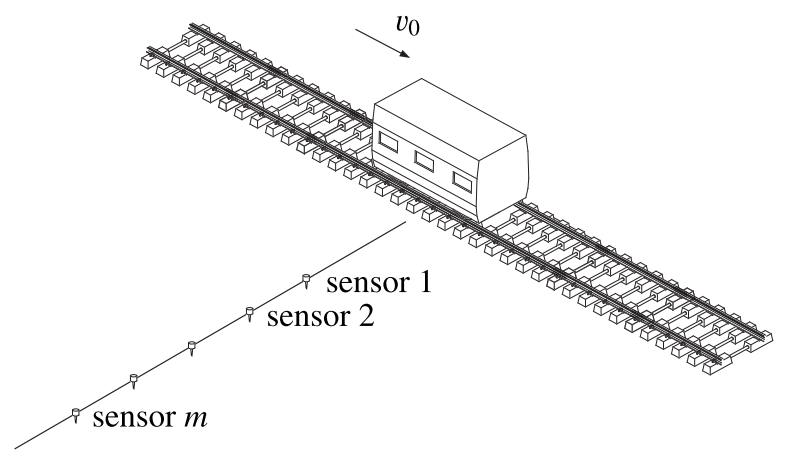

Figure 6. Location of virtual sensors using for the train speed calculation.

where the subscripts $v$ and $t$ are related to the vehicle and to track (including the foundations), respectively. Track stiffness $\mathbf{K}_{t}$, damping $\mathbf{C}_{t}$ and mass $\mathbf{M}_{t}$ matrices are defined with the help of the dynamic parameters of the track in terms of mass, stiffness and damping. $\mathbf{M}_{v}$ is the vehicle mass matrix and $\underline{\mathbf{h}}$ represents a general term gathering among others the contribution of the applied forces. Degrees of freedom are defined by the vector $\underline{\mathbf{q}}_{v}$ (for the vehicle) and by the vector $\mathbf{q}_{t}$ (for the track). For vehicle/track coupling, the wheel/rail contact is defined by non-linear Hertz's contact stress law in force vector $\mathbf{f}_{t}$, implying the non-linear relationship between the imposed load $N$ and the material deformation $d$

$$
N=K_{H z} d^{3 / 2}
$$

where the coefficient $K_{H z}$ depends on the radii of curvature of the wheel and the rail profile, and the elasticity of the material of both bodies. Once the first simulation performed, the time history of the forces exerted by the track on the soil are included to the second model describing the dynamic behaviour of the soil; the simulation of the response of the soil to these forces is performed through a finite element model. The governing equations of motion of the soil subsystem can be written

$$
\mathbf{M}_{s} \underline{\ddot{q}}_{s}+\left(\mathbf{C}_{s}+\mathbf{C}_{d}\right) \underline{\mathbf{q}}_{s}+\mathbf{K}_{s} \underline{\mathbf{q}}_{s}=\underline{\mathbf{f}}_{s o i l}
$$

where $\underline{\mathbf{q}}_{s}$ is the vector related to the degrees of freedom of the soil subsystem. $\mathbf{K}_{s}$ and $\mathbf{M}_{s}$ are the soil mass and stiffness matrices, respectively, and $\mathbf{C}_{s}$ the intrinsic damping matrix, representing the soil viscous damping contribution, which is defined as

$$
\mathbf{C}_{s}=\beta \mathbf{K}_{s} .
$$

Due to the high memory allocations and CPU-times, the studies are limited to small regions of interest (model diameters up to $50-60 \mathrm{~m}$ ).

The first step is based on the philosophy adopted by the train constructor (multibody modelling). Although the rail is modelled with a discretely supported flexible beam, other track parts are considered as lumped masses (sleepers, foundation) with interconnection elements (railpads, ballast). Ground wave propagation and the time history of ground velocities are obtained through the finite element soil model, considered as layered if necessary. Various effects are analysed, in order to verify the method robustness face to the several internal or external parameters. In order to be concise, only the vertical vibration component is retained for the analysis, since the vibration direction does not influence the train speed calculation accuracy [14].

\subsection{Effect of the soil layering}

Soil configuration has a strong influence in mid- and highfrequency ranges, due to the damping attenuation and the magnitude changes induced by the ground layering [15]. Indeed, an exponential decrease of vibration magnitude with distance and an attenuation at high frequencies are observed, mainly due to the damping. By analysing the vibration transmission between the two top soil layers, a resonance occurs with a corresponding frequency defined by $[17,18]$

$$
f_{\text {res }}=\frac{c_{P}}{4 h}
$$

where $c_{P}$ is the longitudinal wave velocity and $h$ the depth of the soil first layer.

The purpose of this section is to emphasise the consideration of the layers in the generated ground vibration data and, more precisely the influence of the soil resonance on the vibration transmission. In practice, an amplification of magnitude is observed around this frequency with a light attenuation in the low frequencies [13]. These considerations are observed in Fig. 8 showing the spectral content of ground vibration at $10 \mathrm{~m}$ from the track due to the passing of a Thalys HST running at $v_{0}=300 \mathrm{~km} / \mathrm{h}$ for homogeneous and layered soil cases. Peak picking from the dominant frequency method is also displayed (train speed estimations at each dominant frequency is shown). By 

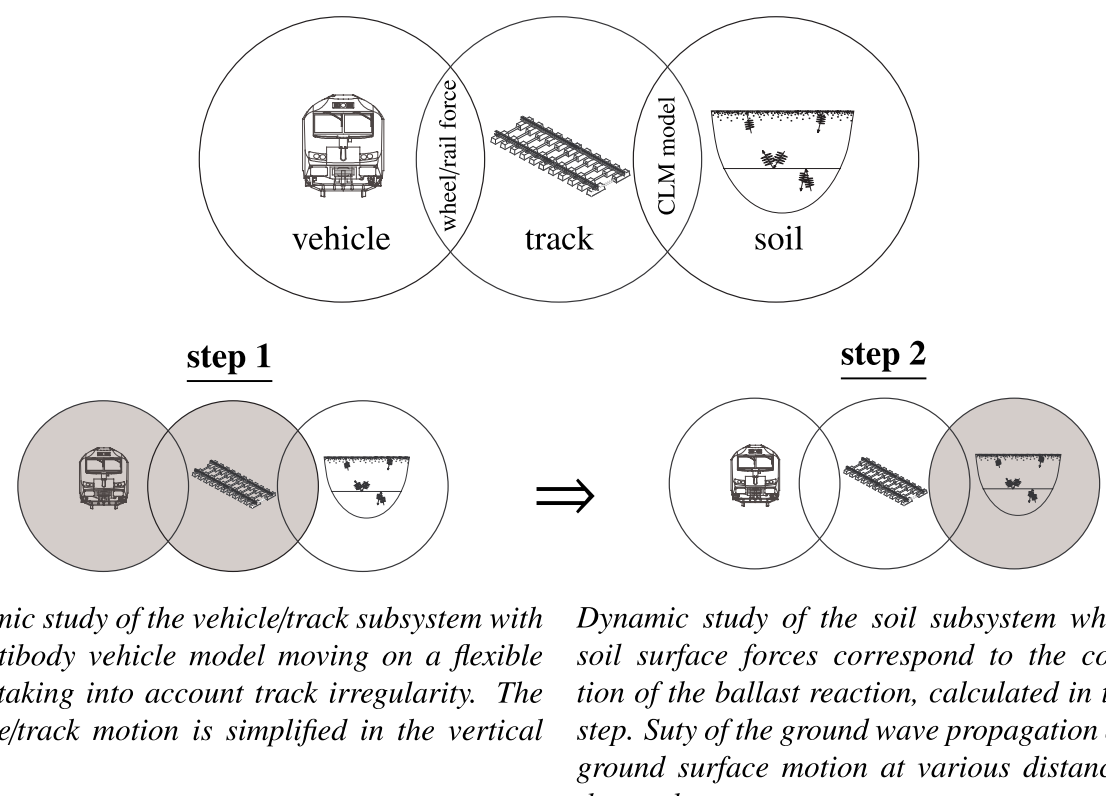

\begin{abstract}
Dynamic study of the vehicle/track subsystem with a multibody vehicle model moving on a flexible track taking into account track irregularity. The vehicle/track motion is simplified in the vertical plane.
\end{abstract}

Dynamic study of the soil subsystem where the soil surface forces correspond to the contribution of the ballast reaction, calculated in the first step. Suty of the ground wave propagation and the ground surface motion at various distance from the track.

Figure 7. Two-step approach model layout used for obtaining numerical ground vibration data.

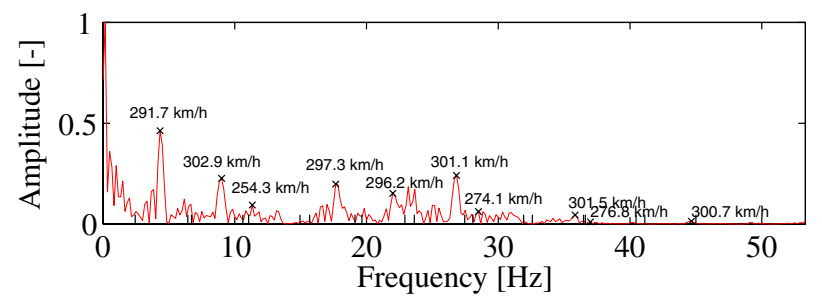

(a) Homogeneous soil (estimated speed: $298.1 \mathrm{~km} / \mathrm{h}$ )

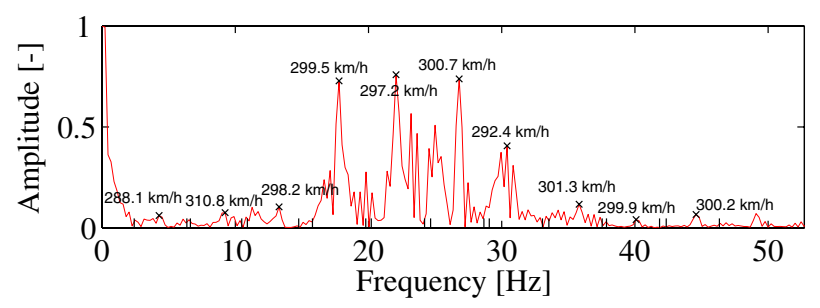

(b) Multi-layered soil (estimated speed: $299.7 \mathrm{~km} / \mathrm{h}$ )

Figure 8. Dominant frequency method applied to the Thalys HST running at $v_{0}=300 \mathrm{~km} / \mathrm{h}$ as a function of the soil configuration.

comparing the two cases, an amplification around $20 \mathrm{~Hz}$ is observed for the layered case, as expected by Eq. (17). Regarding the calculation of vehicle speed, no notable difference appears between the two examples, except a slight improvement for layering appearance, showing that the proposed method is not affected by the soil conditions.

\subsection{Effect of the sensor response}

When measuring ground vibration, the dynamic behaviour of the sensors can affect the response to a mechanical input. Although seismic accelerometers present a relatively uniform and flat response to ground vibrations, this is not the case for velocity-type seismic pickups for which the linear range starts from few $\mathrm{Hz}$, including phase errors.
Table 2. Specification of the studied geophones [19,20].

\begin{tabular}{lcc}
\hline & SM-6 & GS-20DM \\
\hline \hline Natural frequency & $4.5 \mathrm{~Hz}$ & $14 \mathrm{~Hz}$ \\
Spurious frequency & $>140 \mathrm{~Hz}$ & $>400 \mathrm{~Hz}$ \\
Damping ratio & 0.56 & 0.70 \\
Intrinsic sensitivity & $28.8 \mathrm{~V} / \mathrm{m} / \mathrm{s}$ & $17,7 \mathrm{~V} / \mathrm{m} / \mathrm{s}$ \\
Moving Mass & $11.1 \mathrm{~g}$ & $7.8 \mathrm{~g}$ \\
Maximum coil excursion & $4 \mathrm{~mm}$ & $3 \mathrm{~mm}$ \\
\hline
\end{tabular}

The frequency response of a geophone can be defined by its Laplace representation

$$
H(p)=G \frac{p^{2}}{p^{2}+2 \xi \omega_{0} p+\omega_{0}^{2}}
$$

where $G$ is the sensor sensitivity and $\omega_{0}$ and $\xi$ are the natural circular frequency and the damping ratio, respectively. The reader can see that Eq. (18) defines a second-order Butterworth high-pass filter. Its implementation of the numerical signal to analyse is made using the bilinear (or Pade) transformation

$$
p=2 f_{s} \frac{z-1}{z+1}
$$

which allows a smooth analogue-to-digital filter conversion into the digital plane (z-plane), without spectral covering ( $f_{s}$ is the sampling frequency). An important analysis is to quantify the sensitivity of train speed calculator face to pickup response. Two geophones are studied thereafter, differing from their natural frequency defining the filter cut-off frequency (Table 2): one at $4.5 \mathrm{~Hz}$ (geophone SM-6), the other at $14 \mathrm{~Hz}$ (geophone GS-20DM).

Figure 9 shows the corresponding relative errors issued from train speed calculation for both geophone response implementations on numerical results, including the case 


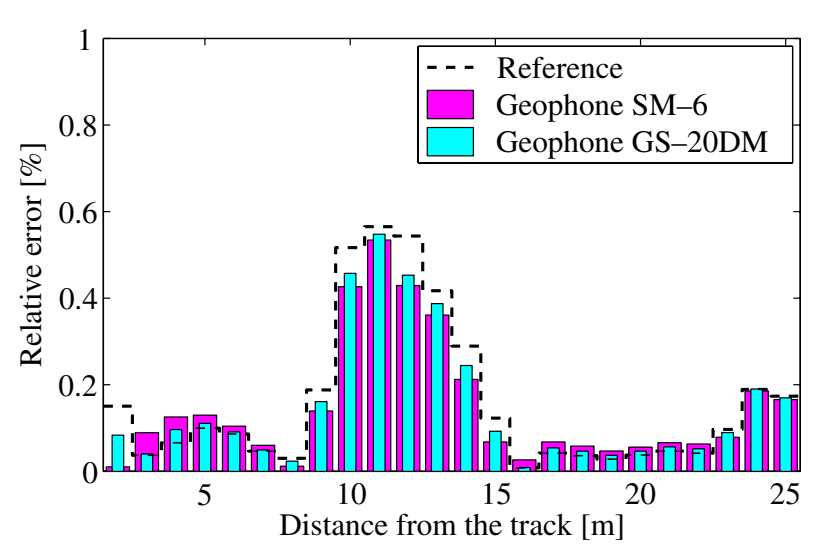

Figure 9. Evaluation differences of the dominant frequency method for the Thalys HST running at $v_{0}=300 \mathrm{~km} / \mathrm{h}$ as a function of the geophone type.

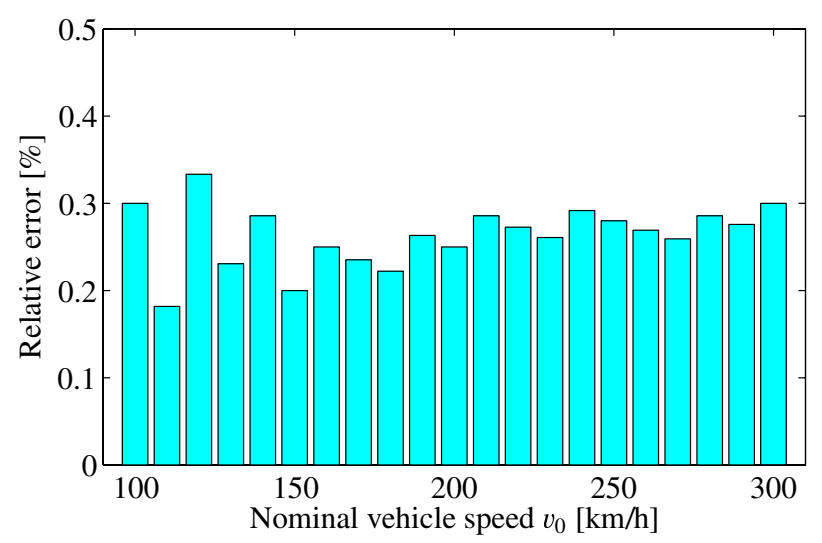

Figure 10. Evaluation differences of the dominant frequency method for the Thalys HST as a function of the speed.

where the sensor response is not retained (reference case). No significant difference is observed, showing that the calculation method is based on the complete usable frequency range (here, up to $50 \mathrm{~Hz}$ ). This figure illustrates also that the location of the sensor (distance from the source) does not affect the accuracy (error less than 1\%).

\subsection{Effect of the vehicle speed range}

Many of the practical limitations could be addressed, one of these being the speed range validity. Figure 10 displays the relative error for a Thalys running at speeds from 100 to $300 \mathrm{~km} / \mathrm{h}$. As multiple ground vibration virtual signals are used, an occurrence analysis is performed to estimate the mean speed. Clearly, no notable influence in magnitude of speed is observed in the results: the relative error less than $0.5 \%$ and without particular correlation with the vehicle speed.

\subsection{Effect of the wheel/rail defect}

Urban networks are characterised by a low speed and a relatively high density of singular rail and wheel surface defects (rail joints, wheel flat, out-of-roughness, ...). The frequency range of track deflection and therefore of ground vibration is very limited due to the low speed. The main contribution of ground vibration comes from

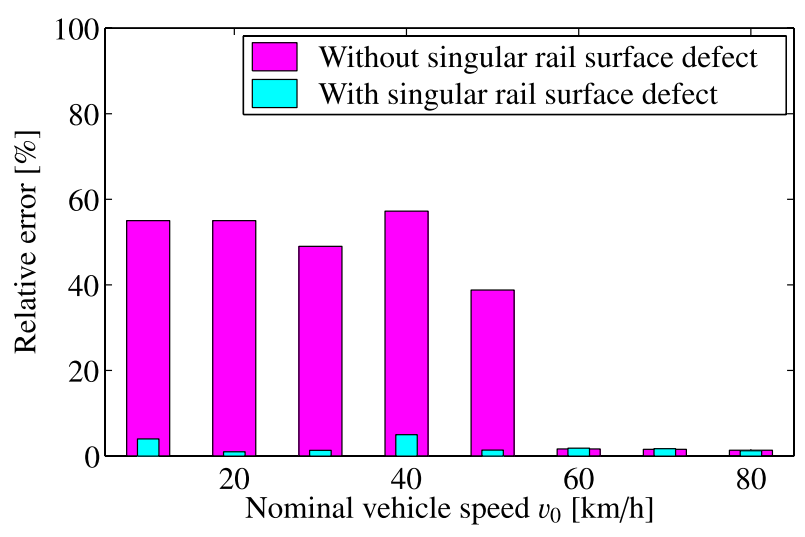

Figure 11. Evaluation differences of the dominant frequency method for the T2000 tram as a function of the speed.

the dynamic wheel/rail interaction [15]. Regarding the modelling, a random axle load cannot be retained because it is presented as a hypothesis of the perfect contact between the rail and the wheel. Physically, these bodies are not rigid, and the reason why they infiltrate each other is because of the train's static and dynamic forces. In the authors' opinion, a detailed model definition starts with the wheel/rail contact and the present model includes the Hertz's contact stress law for more faithfully representing the wheel/rail interaction. Two defects are analysed, commonly encountered in practice, and mainly contributing to the ground wave generation.

Figure 11 compares the effect of a rail joint presence on the speed calculation accuracy. A tram operating in Brussels is studied and Ref. [21] provides additional details about the rail joint modelling. Regarding the ability of the calculation method to estimate train speed, a strong influence is observed for the very low speed with high errors for the absence of defects (while noting that a difference of a few $\mathrm{km} / \mathrm{h}$ induces a large relative error in very low speed). From a speed of $60 \mathrm{~km} / \mathrm{h}$, the error diminishes abruptly, showing that the method depends on a sufficient number of harmonics; low speed cases induces a short range of excitation frequencies, according to to the track deflection spectrum [15]. In the case of a singular defect, even very low speeds are accurately estimated. The presence of a rail joint amplifies dominant peaks with strong amplitudes due to the amplification of the each wheel passage effect.

Regarding the effect by a wheel flat, the findings are different. As the wheel surface defect acts periodically, an additional amplitude modulation can be observed in the excitation (wheel/rail contact forces) and therefore on the response (ground motion) [22], in addition to the dynamic deflection. This effect induces an increase of estimation errors but it is relatively small compared to the errors making without surface defect (Fig. 12).

\subsection{Effect of the external sources}

As the track/soil system is considered as linear, the overall vehicle weight does not affect the numerical result. The possible variation in weight of each wheelset has a small influence on the ground vibration frequencies peak magnitude. In reality (experimental vibrations), the 


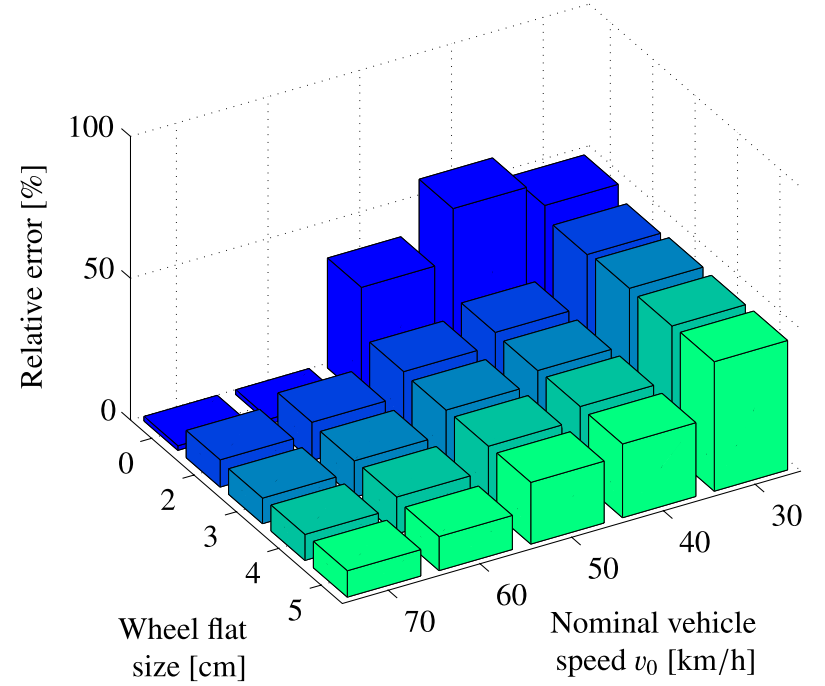

Figure 12. Evaluation differences of the dominant frequency method for the T2000 tram as a function of the speed and of size of the wheel flat.

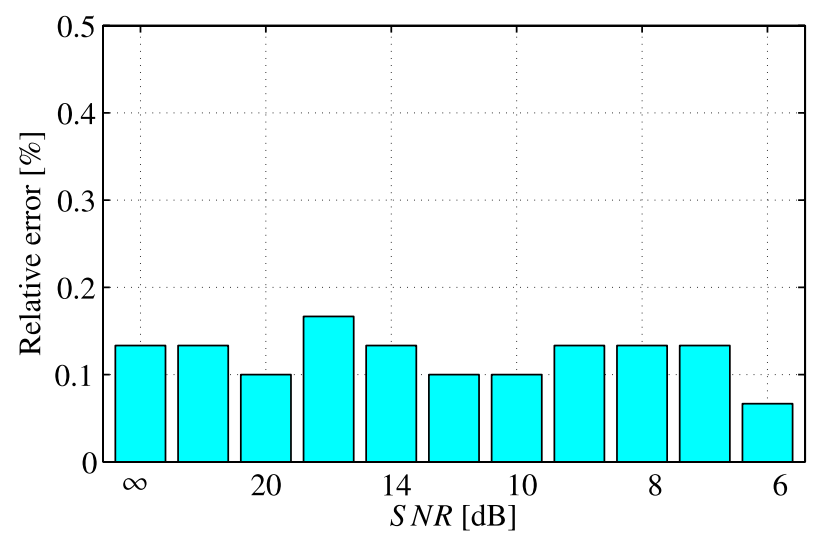

Figure 13. Evaluation differences of the dominant frequency method for the Thalys HST as a function of the signal-to-noise ratio.

background noise affects the method and it is therefore difficult to verify this experimentally. The signal to noise ratio $(S N R)$ is certainly an important parameter influencing the ground vibration spectra and is defined by

$$
S N R=20 \log _{10} \frac{\operatorname{rms}[s(t)]}{\operatorname{rms}[n(t)]}
$$

where $n(t)$ is the noise signal and $s(t)$ the original signal. Numerically, it is possible to create a noisy signal

$$
s_{N}(t)=s(t)+n(t)
$$

by combining these two signals, where $n(t)$ is a normally distributed pseudorandom signal. It allows an estimation of the signal level importance compared to other sources of vibration. Figure 13 presents the calculated relative error for a Thalys HST during at $300 \mathrm{~km} / \mathrm{h}$ when $S N R$ varies and shows the robustness of the proposed calculation method face to external sources.
Table 3. Speed evaluation during the passing of HST's.

\begin{tabular}{cccc}
\hline $\begin{array}{c}\text { Train } \\
\text { type }\end{array}$ & Track & $\begin{array}{c}\# \\
\text { carriages }\end{array}$ & $\begin{array}{c}\text { Calculated speed } \\
{[\mathrm{km} / \mathrm{h}]}\end{array}$ \\
\hline \hline Thalys & $\mathrm{B}$ & 8 & 291.9 \\
Thalys & $\mathrm{A}$ & 8 & 301.8 \\
Thalys & $\mathrm{B}$ & 8 & 298.9 \\
Thalys & $\mathrm{A}$ & 8 & 300.2 \\
Thalys & $\mathrm{A}$ & $2 \times 8$ & 293.2 \\
Eurostar & $\mathrm{A}$ & 18 & 298.9 \\
Thalys & $\mathrm{A}$ & 8 & 288.4 \\
Thalys & $\mathrm{B}$ & 8 & 285.3 \\
Thalys & $\mathrm{B}$ & 8 & 258.5 \\
Eurostar & $\mathrm{B}$ & 18 & 296.9 \\
Thalys & $\mathrm{B}$ & $2 \times 8$ & 297.4 \\
Thalys & $\mathrm{A}$ & 8 & 283.8 \\
Thalys & $\mathrm{A}$ & 8 & 282.7 \\
Thalys & $\mathrm{B}$ & 8 & 302.0 \\
TGV & $\mathrm{A}$ & 8 & 292.4 \\
\hline
\end{tabular}

Table 4. Speed evaluation during the passing of HST's on the high speed line HS1 in UK.

\begin{tabular}{llcc}
\hline Recorded train & Track & Time & Calculated speed $[\mathrm{km} / \mathrm{h}]$ \\
\hline \hline Eurostar & A & $12: 09$ & 261.3 \\
Javelin 395 & B & $12: 10$ & 220.4 \\
Javelin 395 & B & $12: 42$ & 216.2 \\
Javelin 395 & A & $12: 51$ & 223.3 \\
Eurostar & A & $12: 52$ & 285.2 \\
Eurostar & A & $13: 11$ & 289.0 \\
Javelin 395 & A & $13: 21$ & 223.2 \\
Eurostar & A & $13: 25$ & 287.7 \\
Eurostar & A & $13: 41$ & 298.2 \\
\hline
\end{tabular}

\section{Field trials performed on operational railway lines - Applicability of the method to experimental data}

The train speed calculator was used to predict train speeds collected by [13]. In this work, experimental results were used to validate the aforementioned ground vibration prediction model near the high-speed line LGV1 between Brussels and Paris/London. Sensors were placed from 3 to $43 \mathrm{~m}$ from the external side of closest track. Train passages in both track directions were measured. Five-teen train passages were recorded (12 Thalys, 2 Eurostar HST and 1 French TGV). The Eurostar TransManche super train had the same configuration as the Thalys HST (Fig. 14), except in the centre where two side carriages are added for safety reasons related to tunnel passage. Its length was nearly twice than the Thalys with a total dimension of $394 \mathrm{~m}$. The French TGV is currently the forerunner HST and mainly circulates in France. It has the same dimensions as the Thalys (Fig. 14). It should be mentioned that "Thalys double" passages were also recorded. These consisted of two single Thalys trains hooked up in series. Table 3 lists each passage with the vehicle speed calculated by the present method.

More recently, Connolly performed a similar experimental analysis for the purpose of analysing the effect 


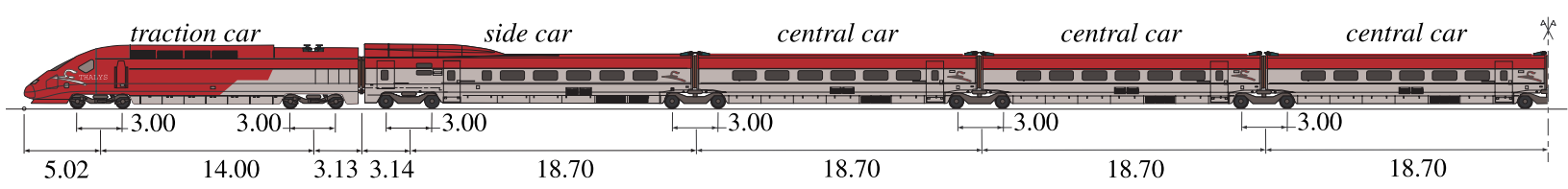

Figure 14. Thalys HST dimensions.

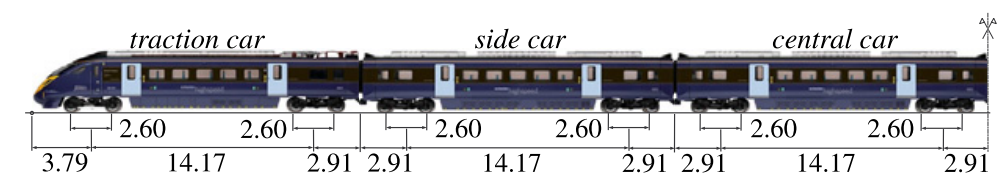

Figure 15. Javelin 395 HST dimensions.

of embankment conditions on high speed rail ground vibrations in UK. High Speed 1 (HS1), linking London, UK and Paris, France via the Channel Tunnel beneath the English Channel, has been investigated. Passages of Eurostar and Javelin HST's were recorded on $25^{\text {th }}$ September 2012 at a test site close to Hollingbourne, UK. The Javelin Class 395 is an electric multiple unit built in Japan by Hitachi for high speed commuter services on HS1. It is capable of running at a maximum speed of $225 \mathrm{~km} / \mathrm{h}$ under overhead electrification on HS1, and $161 \mathrm{~km} / \mathrm{h}$ on $750 \mathrm{~V}$ DC third rail supply on conventional lines. Similarly to Thalys trains, two Javelings can be linked together. Therefore the basic 6-carriage train can be adapted to create a 12-carriage system. Javelin class 395 trains are composed of intercity train carriages that have been upgraded to facilitate elevated speeds. Therefore more conventional bogies are used (for all carriages) in comparison to dedicated high speed bogies found in Thalys, TGV and Eurostar trains (Fig. 15). Unlike the aforementioned high speed trains, the Javelin carriage spacing $\left(L_{c}=20 \mathrm{~m}\right)$ is greater than the bogie spacing $\left(L_{b}=14.17 \mathrm{~m}\right)$. Therefore the excitation frequencies associated with $L_{b}$ and $L_{c}$ are different. Table 4 lists the different passages recorded and the calculated vehicle speed. Other speed calculations have been performed and are been used for an experimental analysis of groundborne vibration levels generated by high speed rail lines on various earthwork profiles [23].

\section{Conclusions}

Ground vibrations generated by the passing of a train can be seen as an opportunity to capture the train speed. Based on the dominant frequency method, a tool is presented in this paper. Various signal processing procedures are implemented in order to offer a semi-remote, non-invasive and economical method for vehicle speed monitoring. In the case of ground vibration measurement, no additional device is needed.

To show the robustness and ability of the proposed method to calculate a wide range of train speeds, it is used to predict speeds from numerically generated train passages. Several factors are analysed at different levels of applicability to varying circumstances. It is found that a low excitation frequency range can influence accuracy and that additional excitations (caused by vehicle or soil parameters) improve its performance. The method is then applied to measured results in order to complete an experimental database useful for further numerical prediction model validation.

\section{References}

[1] D.J. Thompson, Railway noise and vibration. Mechanisms, modelling and means control (Elsevier, Oxford (UK), 2009)

[2] C.J. Taylor, Railway Gazette International 009/07, 553 (2007)

[3] C. Madshus, A.M. Kaynia, Journal of Sound and Vibration 231, 689 (2000)

[4] G.Degrande, L. Schillemans, Journal of Sound and Vibration 247, 131 (2001)

[5] P.Galvín, J. Domínguez, Soil Dynamics and Earthquake Engineering 29, 641 (2009)

[6] L. Auersch, Journal of Rail and Rapid Transit 226, 469 (2012)

[7] D.P. Connolly, G. Kouroussis, A. Giannopoulos, O. Verlinden, P.K. Woodward, M.C. Forde, Soil Dynamics and Earthquake Engineering 58, 37 (2014)

[8] B. Picoux, R. Rotinat, J.P. Regoin, D.L. Houédec, Journal of Sound and Vibration 267, 575 (2003)

[9] B. Picoux, D.L. Houédec, Soil Dynamics and Earthquake Engineering 25, 905 (2005)

[10] S. Iwnicki, Vehicle System Dynamics 30, 295 (1998)

[11] S. Iwnicki, Handbook of railway vehicle dynamics (CRC Press, New York (USA), 2006)

[12] S.H. Ni, Y.H. Huang, K.F. Lo, Computers and Geotechnics 38, 416 (2011)

[13] G. Kouroussis, O. Verlinden, C. Conti, Soil Dynamics and Earthquake Engineering 31, 692 (2011)

[14] G. Kouroussis, D.P. Connolly, M.C. Forde, O. Verlinden, Journal of Rail and Rapid Transit, doi: 10.1177/0954409713515649 (2014)

[15] G. Kouroussis, D.P. Connolly, O. Verlinden, International Journal of Rail Transportation 2, 69 (2014)

[16] G. Kouroussis, L. Van Parys, C. Conti, O. Verlinden, Advances in Engineering Software 70, 63 (2014)

[17] S.L. Kramer, geotechnical earthquake engineering (Prentice-Hall, New Jersey (USA), 1996)

[18] G. Lefeuve-Mesgouez, A.T. Peplow, D.L. Houédec, Soil Dynamics and Earthquake Engineering 22, 459 (2002) 
[19] Geo Space Technologies, Small digital grade geophone, http://www.geospace.com (February 2014)

[20] Input/Output Company, Sensor: Analog geophones, http: //www . iongeo.com (February 2014)

[21] G. Kouroussis, O. Verlinden, C. Conti, Vehicle System Dynamics 48, 1553 (2010)

[22] G. Alexandrou, G. Kouroussis, O. Verlinden, Modelling the effect of wheel flat on railway-induced ground vibrations, in Proceedings of the Second International Conference on Railway Technology: Research, Development and Maintenance, edited by J. Pombo (Civil-Comp Press, Ajaccio (France), 2014)

[23] D.P. Connolly, G. Kouroussis, P.K. Woodward, P.A. Costa, O. Verlinden, M.C. Forde, Soil Dynamics and Earthquake Engineering 67, 102 (2014) 